\title{
Enhanced Electrical Performance and Heat Dissipation in AlGaN/GaN Schottky Barrier Diodes Using Hybrid Tri-anode Structure
}

\author{
Jun Ma, Giovanni Santoruvo, Pulkit Tandon, and Elison Matioli
}

\begin{abstract}
Enhanced performance in AlGaN/GaN Schottky barrier diodes (SBDs) is investigated using a nanowire hybrid tri-anode structure that integrates three-dimensional Schottky junctions with tri-gate transistors. The fabricated SBDs presented an increased output current density with improved linearity, above $1 \mathrm{~A} / \mathrm{mm}$ at $5 \mathrm{~V}$ when normalized by effective anode width, over 3 orders of magnitude lower reverse leakage current and superior heat dissipation. The sidewall Schottky contacts reduced the turn-on voltage and eliminated the non-ideality caused by the AlGaN barrier. The large surface area of tri-gate architecture greatly enhanced heat dissipation and largely reduced the average temperature as well as thermal resistance of the integrated tri-gate transistors. The trench conduction near $\mathrm{SiO}_{2} / \mathrm{GaN}$ interface, formed under forward bias at both sidewalls and bottom of nanowire trenches, compensated part of the self-heating degradation and improved the output linearity of the device. Optimal design for the tri-anode structure, based on a model of critical filling factor, was proposed to surmount the issue of partial removal of two-dimensional electron gas (2DEG), unveiling the potential of nanostructured GaN devices to achieve comparable or even larger output current than counterpart planar devices.
\end{abstract}

Index Terms - AlGaN, GaN, nanowire, tri-gate, tri-anode, self-heating, trench conduction, filling factor, Schottky diode, HEMT.

\section{INTRODUCTION}

$\mathrm{G}$ $\mathrm{aN}$ is one of the most promising candidates for future power electronics. Given the large band-gap, superior breakdown field strength and excellent electron saturation velocity, GaN power devices can operate at high temperature, high voltage and high frequency, enabling efficient and compact power management as compared to the conventional Si technology [1-4]. Among GaN power devices, Schottky barrier diodes (SBDs) are an important member with wide applications in power converters. However, conventional AlGaN/GaN SBDs suffer from high turn-on voltage $\left(V_{\text {on }}\right)$ and large reverse leakage current $\left(I_{R}\right)$. A large effort has been devoted to address these challenges, but common technologies proposed to mitigate on-state (off-state) challenges tend to

J. Ma, G. Santoruvo, P. Tandon and E. Matioli are with the Power and Wide-band-gap Electronics Research Laboratory (POWERlab), École polytechnique fédérale de Lausanne (EPFL), CH-1015 Lausanne, Switzerland (e-mail: jun.ma@epfl.ch; elison.matioli@epfl.ch).

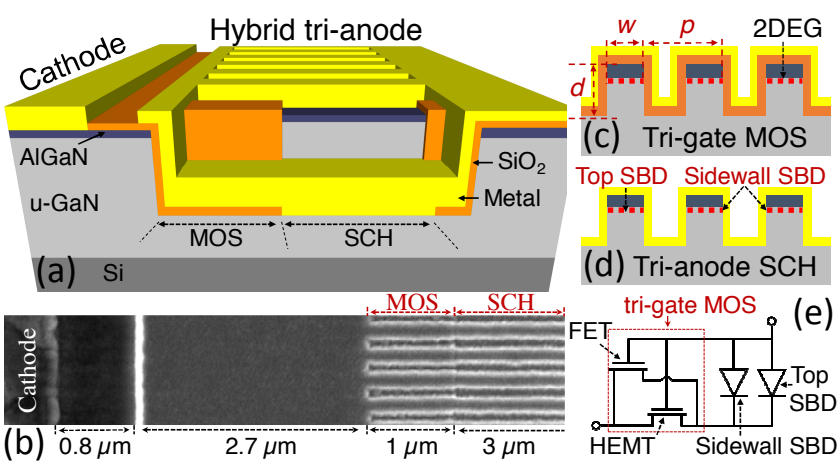

Fig. 1. (a) Schematics of the hybrid tri-anode SBD; (b) top-view SEM observation; cross-sectional schematics for (c) tri-gate MOS and (d) tri-anode Schottky (SCH) regions; (e) equivalent circuit of the hybrid tri-anode SBD.

degrade off-state (on-state) performance [5-7]. In addition, self-heating is another major issue that hinders the performance of GaN SBDs as well as transistors [8], since high temperature degrades significantly the performance, efficiency and reliability of the devices. Engineering of heat dissipation is therefore highly desirable for miniaturization and densification of these devices.

A hybrid tri-anode structure has been recently proposed for AlGaN/GaN SBDs, resulting in reduced $V_{\text {on }}$, improved ideality factor and ultra-low $I_{R}$ [4]. In this work we investigated the electrical enhancement in hybrid tri-anode SBDs and demonstrated further advantages in forward characteristics such as large output current density, better linearity and improved heat dissipation of this architecture. Enhanced output current density (I) with improved output linearity were achieved with a hybrid tri-anode structure due to the sidewall Schottky contact, enhanced heat dissipation and additional trench conduction channels near oxide/GaN interface. Schottky junction formed at nanowire sidewalls reduced the $V_{o n}$ and eliminated the non-ideal behavior observed in planar SBDs. The self-heating was minimized by the nanowire trenches which largely reduced the thermal resistance of the integrated tri-gate transistors as compared to the counterpart planar devices. Conduction channels at the $\mathrm{SiO}_{2} / \mathrm{GaN}$ interfaces were found to form at not only sidewalls but also trench bottoms with increasing forward bias, which improved the output linearity of the device. This observation challenges assumptions from previous studies where only sidewall conduction is present in 
such geometry, as discussed later in this paper. Optimal tri-anode design was discussed based on a critical filling factor model, which could be generally applied to other GaN nanostructure-based approaches such as tri-gate, $\Omega$-gate, Fin-shaped, nanochannel array and multi-mesa-channel transistors [9-14].

\section{DEVICE STRUCTURE}

The $\mathrm{AlGaN} / \mathrm{GaN}$ epi in this work consisted of 3.3- $\mu \mathrm{m}$ buffer,

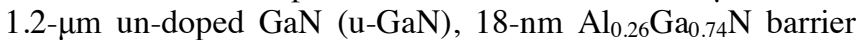
and 2-nm u-GaN cap layers. Schematics and scanning electron microscopy (SEM) observation of the $100-\mu \mathrm{m}$-wide tri-anode SBDs were shown in Fig. 1. The device fabrication started with mesa etching by $\mathrm{Cl}_{2}$-based inductively coupled plasma (ICP), followed by metal deposition (Ti/Al/Ni/Au) and ohmic thermal annealing. The AlGaN/GaN nanowires were defined by interference lithography and ICP etching. The depth $(d)$ of the nanowire etching was $114 \mathrm{~nm}$. The width $(w)$ of a nanowire was $135 \mathrm{~nm}$ and the period $(p)$ was $300 \mathrm{~nm}$, corresponding to a filling factor $(F F=w / p)$ of 0.45 . Then $18-\mathrm{nm} \mathrm{SiO}_{2}$ was deposited by atomic layer deposition and selectively removed in Schottky and contact regions. Finally the anode was formed by deposition of $\mathrm{Ni} / \mathrm{Au}$, integrating three-dimensionally $\mathrm{Ni} / \mathrm{AlGaN} / \mathrm{GaN}$ (top SBD) and $\mathrm{Ni} / \mathrm{GaN}$ (sidewall SBD) Schottky junctions as well as $\mathrm{SiO}_{2} / \mathrm{AlGaN} / \mathrm{GaN}$ tri-gate metal-oxide-semiconductor transistors (tri-gate MOS) to form a hybrid tri-anode. Planar $\mathrm{AlGaN} / \mathrm{GaN}$ SBDs with similar dimensions based on conventional planar MOS and $\mathrm{SCH}$ regions were taken as reference.

\section{RESULTS AND DISCUSSION}

Figure 2 shows the $I$ and conductance $(G)$ versus anode bias $(V)$ of the SBDs with planar (Planar) and hybrid tri-anode (Tri-anode) structures, obtained by normalizing the current with effective anode width $\left(w_{a}\right)$. The sidewall metal-to-2DEG contact in the Tri-anode was considered by defining the $w_{a}$ as $n$ $\times(w+2 b)$, where $n$ is the number of nanowires in a given device and $b$ represents the width of the Ni-to-2DEG sidewall modulation region $(10 \mathrm{~nm})$ [15]. For each of the structures, at least 12 devices were measured, and small error bars in Fig. 2 reveal little variation between devices of the same kind. The Planar exhibited a large $V_{\text {on }}$ of $1.43 \pm 0.11 \mathrm{~V}$ and an undesirable knee voltage. This knee voltage was eliminated in the Tri-anode, with $V_{\text {on }}$ reduced to $0.95 \pm 0.09 \mathrm{~V}$. The on-resistance $\left(R_{o n}\right)$ of the Planar and Tri-anode was $3.38 \pm 0.16$ and $3.48 \pm$ $0.11 \mathrm{ohm} \cdot \mathrm{mm}$, respectively. Furthermore, the Planar presented a resistive linear behavior under small bias, due to the $\mathrm{AlGaN}$ barrier, while the Tri-anode presented $I-V$ characteristics much closer to an ideal diode (the inset of Fig. 2 (b)). The $I_{R}$ was also reduced by over 3 orders of magnitude for the Tri-anode (Fig. 2 (b)). These improvements can be explained by the integrated sidewall SBDs and tri-gate MOS in the Tri-anode. As shown Fig. 1 (e), the equivalent circuit of the hybrid tri-anode consists of Top and Sidewall SBDs as well as the tri-gate MOS. The sidewall SBD has a smaller and close-to-ideal Schottky barrier due to the absence of the AlGaN barrier, which led to a smaller $V_{o n}$ and eliminated the non-ideality observed in the Planar.
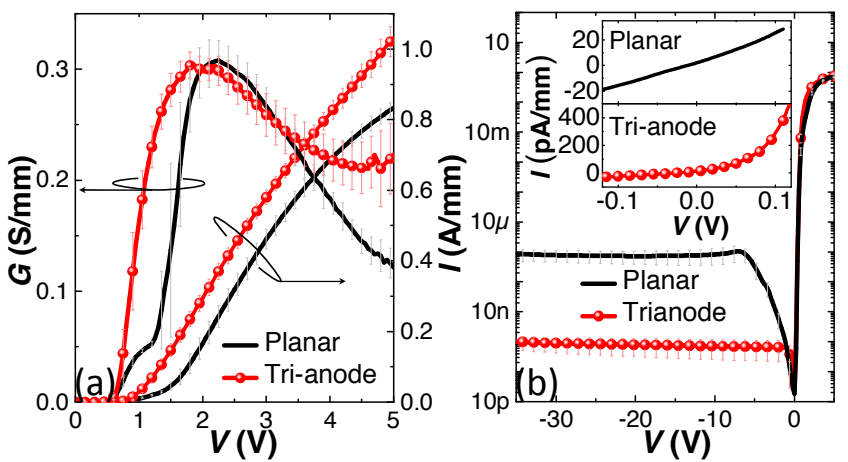

Fig. 2. (a) Forward and (b) reverse electric characteristics of the SBDs; the inset shows the $I-V$ characteristics of the SBDs under small bias.

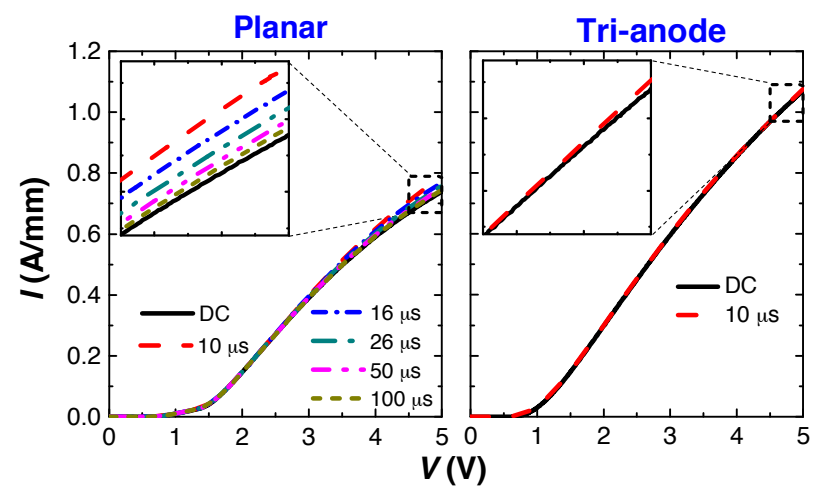

Fig. 3. DC and pulsed $I-V$ characteristics of the SBDs with different pulse widths; the period of the pulse was kept constant as $1 \mathrm{~s}$.
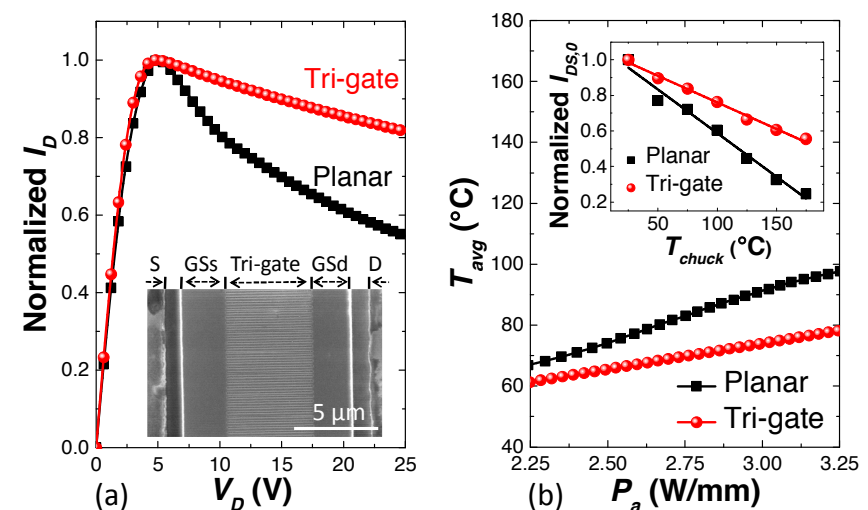

Fig. 4. (a) Normalized $I_{D}-V_{D}$ characteristics of planar and tri-gate AlGaN/GaN MOS transistors measured with similar gate driving voltage $\left(V_{G}-V_{t h}=\sim 5 \mathrm{~V}\right)$ and (b) extracted average temperature of the $150-\mu \mathrm{m}$-wide transistors versus applied power normalized by device width; the insets of (a) and (b) show the SEM observation of the tri-gate transistor and the linear dependences of the extrapolated saturation drain current on the chuck temperature, respectively.

Under reverse bias, the tri-gate MOS was depleted when $V$ was below its threshold voltage $\left(V_{t h}\right)$, largely suppressing the $I_{R}$. The reduction of $I_{R}$ depends on $V_{t h}$ of the tri-gate MOS [4], which can be controlled by $w$ and the thickness of the $\mathrm{SiO}_{2}[11,16]$.

Fig. 2 (a) also reveals a more linear $I-V$ characteristics with the Tri-anode. The first explanation for this is the minimized self-heating of the Tri-anode. To explore this, DC as well as pulsed I-V characteristics of the samples are compared in Fig. 3. An increase in current with reduced pulsed widths from 100 to $10 \mu \mathrm{s}$ was observed for the Planar, indicating self-heating degradation of its DC performance. There was no obvious difference in DC and pulsed $I-V$ characteristics for the 
Tri-anode even with a pulse width down to $10 \mu \mathrm{s}$ (the shortest pulse width possible with our measurement equipment) and current over $1 \mathrm{~A} / \mathrm{mm}$. These results suggest a significant reduction of self-heating in the Tri-anode likely due to the improved heat dissipation from the nanowire trenches with large metal surface area, resulting in less thermal degradation in conductance of the tri-gate MOS. To verify this, AlGaN/GaN tri-gate high electron mobility transistors (HEMTs) with similar nanowires to the Tri-anode were compared with planar MOS transistors, and their normalized drain current-voltage $\left(I_{D^{-}} V_{D}\right)$ characteristics are shown in Fig. 4 (a). Using the technique proposed in Ref. [17], we measured the temperature dependence of the extrapolated drain saturation current $\left(I_{D S, 0}\right)$ and estimated the average temperature over the entire device active area $\left(T_{\text {avg }}\right)$ as shown in Fig. 4 (b) (as justified in Ref. [18], the $T_{\text {avg }}$ obtained by this method is close to the $T_{\text {avg }}$ measured by micro-Raman thermography at low power levels although it may neglect the effect of traps). With the same applied power $\left(P_{a}\right)$, the tri-gate HEMT exhibited a much reduced $T_{a v g}$ compared to the planar, which is comparable or greater than other technologies proposed for thermal management of $\mathrm{GaN}$ electronics such as graphene-graphite quilts [19], $\mathrm{Cu}$-filled backside via [20], substrate transfer using h-BN [21] and nanocrystalline diamond thin film [22]. Figure 4 (b) also suggests a smaller thermal resistance $\left(R_{T H}\right)$ for the tri-gate HEMT, which is consistent with a recent report in the literature [10]. From the slope of the linear region of the $T_{a v g}$ versus $P_{a}$ curves, $R_{T H}$ of the planar and tri-gate HEMTs were estimated to be about 34.2 and $17.0 \mathrm{~K} \cdot \mathrm{mm} / \mathrm{W}$, respectively, normalized by device width. The reduction in $R_{T H}$ of about $50 \%$ in the tri-gate HEMT is very close to the reduction $(55 \%)$ by replacing the $\mathrm{Si}$ substrate with $\mathrm{SiC}$ [23], indicating the great potential of the tri-gate technology in thermal engineering of GaN transistors. The reduced $R_{T H}$ in the tri-gate HEMT can be attributed to mainly two reasons: $i$. the increased surface area with nanowire architecture and ii. the absence of the AlGaN barrier at the sidewalls, since AlGaN has much lower thermal conductivity with respect to $\mathrm{GaN}$ [24], which improves heat dissipation through the surface. The reduced $T_{a v g}$ and $R_{T H}$ confirm the improved heat dissipation in the tri-gate HEMT, which is consistent with the results from pulsed measurements for the tri-anode SBDs.

Besides the enhanced heat dissipation, another reason for the better output linearity of the Tri-anode is the so-called sidewall conduction, which forms additional channels at sidewall $\mathrm{SiO}_{2} / \mathrm{GaN}$ interface as a field effect transistor (FET) in parallel with the HEMT and reduces the resistance of the tri-gate MOS (Fig. 1(e)). The sidewall conduction was suggested by previous studies [25-28] but has not been investigated, in addition carrier accumulation at the oxide/GaN interface at trench bottom of the nanowires was neglected. To investigate this effect, periodically gate-recessed MOS transistors were fabricated (Fig. 5 (a) and (b)). Gate length of the transistors was $132 \mu \mathrm{m}$ and the gate-to-source/gate-to-drain distance was $4 \mu \mathrm{m}$. The gate region of the transistors was periodically recessed with a depth of $114 \mathrm{~nm}$ and a length of $165 \mathrm{~nm}$ (period was $300 \mathrm{~nm}$ ). Transfer characteristics of the fabricated transistor (Fig. 5 (c)) reveal current flows perpendicularly to the nanowires with normally-off behavior, indicating that carriers accumulated at

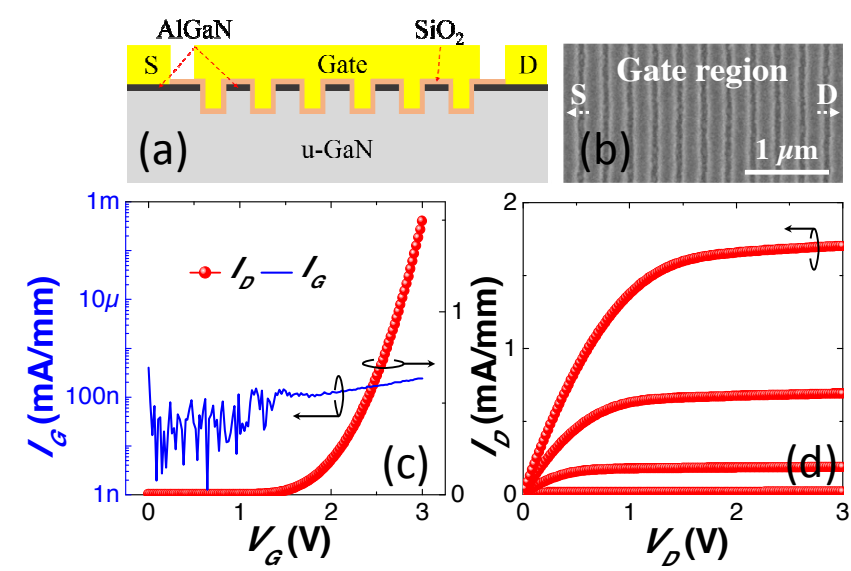

Fig. 5. (a) Cross-sectional schematic and (b) top-view SEM observations in gate region of the periodically gate-recessed transistors; (c) transfer and (d) output characteristics of the transistors.

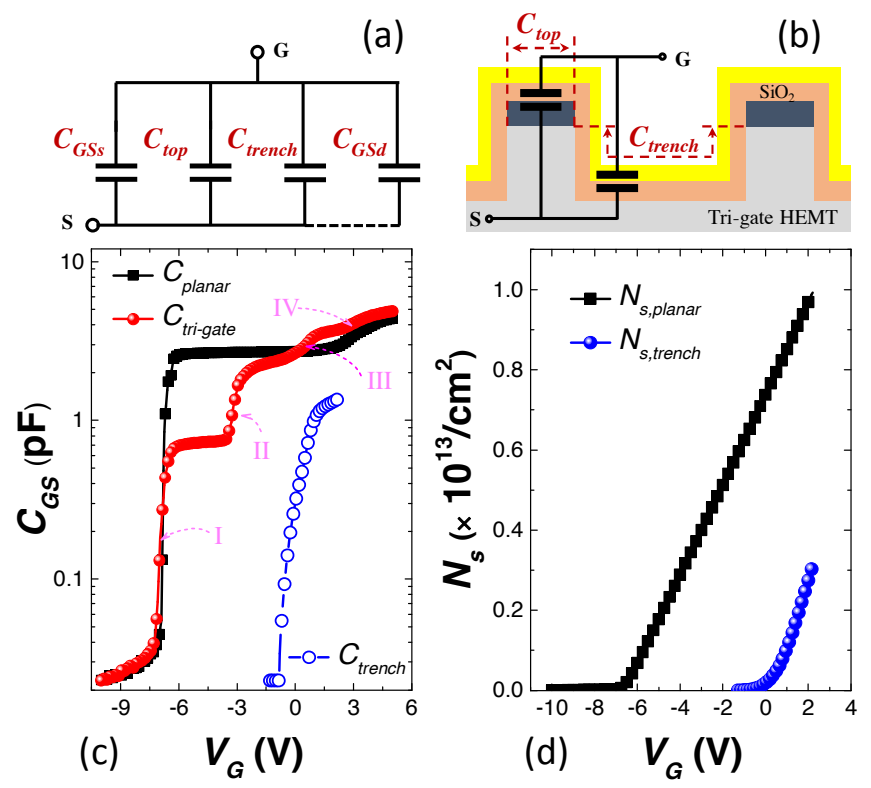

Fig. 6. (a) Equivalent circuit of $C_{G S}$ for the tri-gate HEMT; (b) schematic of $C_{\text {top }}$ as well as $C_{\text {trench }}$ in the tri-gate region; (c) measured $C$ - $V$ characteristics of the HEMTs and extracted $C_{\text {trench }}$; (d) $V_{G}$-dependent sheet carrier density of the planar HEMT and the trench conduction.

the sidewalls as well as the bottom of each trench, and the two accumulation regions overlapped with each other with a threshold voltage of $\sim 2 \mathrm{~V} . I_{D^{-}} V_{D}$ plots of the transistors are presented in Fig. 5 (d), showing that accumulation-induced channels were well modulated by the gate voltage. These results clearly show that carrier accumulation at $\mathrm{SiO}_{2} / \mathrm{GaN}$ interface forms conduction channels in the trenches and contributes to the output current of the Tri-anode. This contribution would increase with larger bias, compensating self-heat degradation and improving output linearity of the Tri-anode.

To investigate the contribution of the trench conduction, gate-source capacitance $\left(C_{G S}\right)$ of the tri-gate and planar HEMTs were studied, denoted as $C_{\text {tri-gate }}$ and $C_{\text {planar }}$, respectively. The $150-\mu \mathrm{m}$-wide tri-gate HEMT had similar nanowires to the Tri-anode in which the direction of the current was parallel to the nanowire. Gate lengths of the HEMTs were both $10 \mu \mathrm{m}$ (the 
inset of Fig. 4 (a)). $C_{\text {tri-gate }}$ is composed of four capacitors in parallel as shown in Fig. 6 (a). The first capacitor is the $\mathrm{SiO}_{2} / \mathrm{AlGaN} / \mathrm{GaN}$ top gates in the nanowire region $\left(C_{\text {top }}\right)$, and the second is the $\mathrm{SiO}_{2} / \mathrm{GaN}$ in nanowire trenches $\left(C_{\text {trench }}\right)$, which acted similarly to a field effect transistor (FET), as shown in Fig. 6 (b). The other two capacitors are the $\mathrm{SiO}_{2} / \mathrm{AlGaN} / \mathrm{GaN}$ planar potions of the gate, one close to the source $\left(C_{G S s}\right)$ and the other close to the drain $\left(C_{G S d}\right)$, corresponding to the GSs and GSd regions in the inset of Fig. 4 (a). Figure. 6 (c) shows the $C$ - $V$ characteristics of the tri-gate and planar HEMTs. The steps I-IV observed for the $C$ - $V$ characteristics of the tri-gate HEMT corresponded to carrier accumulation in $C_{G S s}, C_{\text {top }}+C_{G S d}, C_{\text {trench }}$ and at the $\mathrm{SiO}_{2} / \mathrm{AlGaN}$ interface, respectively. $C_{t o p}$ showed a less negative $V_{t h}$ with respect to $C_{G S s}$ due to sidewall gate depletion and partial relaxation of $\mathrm{AlGaN} / \mathrm{GaN}$ nanowires [29]. Before step IV, $C_{\text {tri-gate }}$ is simply the sum of $C_{G S s}, C_{\text {top }+G S d}$ and $C_{\text {trench }}$. Assuming that these capacitors shared similar values of capacitance in depletion, $C_{\text {trench }}$ was extracted using $C_{\text {trench }}=C_{\text {tri-gate }}-\left[C_{G S s}+\right.$ $\left(C_{\text {top }}+C_{G S d}\right)$ ] when $V_{G}$ was below $2 \mathrm{~V}$ since i. there was no spillover; ii. these capacitor were in parallel; iii $C_{G S s}+\left(C_{t o p}+\right.$ $C_{G S d}$ ) was saturated and its value was close to the value of $C_{\text {tri-gate }}$ before the $V_{T H}$ of $C_{\text {trench }}$, as shown in Fig. 6 (c). Figure 6 (d) presents the comparison between the sheet carrier density of the planar HEMT $\left(N_{s, p l a n a r}\right)$ and the trench $\left(N_{s, t r e n c h}\right)$, extracted from the $\mathrm{CV}$ measurements. $N_{\text {s,trench }}$ is the equivalent carrier density obtained by normalizing the number of carriers with the product of trench length and width (not considering the four sidewalls of the trench) for a fair comparison with the planar HEMT. At a $V_{G}$ of $2 \mathrm{~V}$, the $N_{\text {s,trench }}$ was about $28.4 \%$ of the $N_{s, p l a n a r}$, which represents a significant portion of the carrier density and should not be neglected.

Besides the carrier density, electron mobility $(\mu)$ is another important factor that impacts the current in the trench. According to Ref [30,31], the voltage-dependent effective mobility $\left(\mu_{\text {eff }}\right)$ of $\mathrm{SiO}_{2}$ /etched GaN channel is only about 16 $\mathrm{cm}^{2} / \mathrm{V} \cdot \mathrm{s}$. This value is much lower than the low-field electron mobility $\left(\mu_{0}\right)$ and $\mu_{\text {eff }}$ of the planar HEMT equal to 1560 and $700 \mathrm{~cm}^{2} / \mathrm{V} \cdot \mathrm{s}$ at $V_{G}=2 \mathrm{~V}$, respectively, extracted using a mobility-degradation model $[32,33]$. The $\mu_{0}$ was close to the Hall mobility, which was about $1660 \mathrm{~cm}^{2} / \mathrm{V} \cdot \mathrm{s}$. Since the drain current of a transistor is proportional to the product of carrier density and $\mu$, the drain current of the FET was about $6.5 \%$ of the counterpart planar HEMT at $V_{G}=2 \mathrm{~V}$. With increasing gate bias, this portion will further increase because of two factors: i. the slope of the $N_{s, t r e n c h}-V_{G}$ curve is larger with respect to the $N_{s, p l a n a r}-V_{G}$ curves and ii. the $\mu_{\text {eff }}$ of the FET doesn't change too much with increasing $V_{G}$ [30] while that of the planar HEMT keeps degrading [34]. Consequently, the contribution of trench conduction to the output current of the Tri-anode is then estimated to be small but not negligible, which compensated partly the thermal degradation under high bias and even slightly impacted the on-resistance under low bias. Analogous to normally-off $\mathrm{AlGaN} / \mathrm{GaN}$ transistors with oxide/GaN gate region, the key factor that limits the trench conduction is the low electron mobility due to the etching-induced damages at the $\mathrm{SiO}_{2} / \mathrm{GaN}$ interface [35]. Such damages create a nitrogen-deficient surface and introduce high-concentration donor states, which greatly increase the probability of Coulomb scattering and hence degrade the electron mobility [30,36-37]. However, this mobility can be possibly enhanced by a few means. The first way is to improve the crystalline quality of $\mathrm{GaN}$. For instance, 4-times higher electron mobility has been achieved for dry-etched $\mathrm{GaN}$ on sapphire [38] with respect to that on $\mathrm{Si}[30,31]$. Secondly, optimized etching and recovery processes can also be helpful in reducing such damage. These methods would enhance the trench conduction and correspondingly that of the tri-gate HEMT or the Tri-anode. Furthermore, it should also be noted that, although the contribution of the trench conduction was small in this work, its impact can be relatively increased and possibly even dominating for other tri-gate or nanowire devices with very small $F F$ or very narrow $\mathrm{AlGaN} / \mathrm{GaN}$ nanowires in which the 2DEG is almost fully depleted.

The hybrid tri-anode structure improved the performance of the SBD in many aspects, but it sacrifices $~ 55 \%$ of the $2 \mathrm{DEG}$ due to the nanowire etching $(F F=0.45)$. This reduction in carriers can be compensated by increasing the $F F$. Figure 7 (a) shows the current densities $I_{d}$ and $I_{e d}$ obtained by two normalizations: i. by device width $\left(w_{d}=100 \mu \mathrm{m}\right)$ and ii. by effective device width $\left(w_{e d}=n \times w\right.$ for Tri-anode and $w_{e d}=100$ $\mu \mathrm{m}$ for Planar). While $I_{e d}$ was larger for the Tri-anode, the corresponding $I_{d}$ became smaller than that of the Planar, because in this case the current was normalized by both widths of nanowires (with 2DEG) and trenches (without 2DEG). The absolute current of a single nanowire in Tri-anode is $9.05 \times 10^{-5}$ A at $V=3 \mathrm{~V}$ using $I_{\text {ed,trianode }} \times w$. To achieve the same current at $V=3 \mathrm{~V}$ using the Planar, a width of $\sim 197 \mathrm{~nm}\left(w_{1}\right)$ is needed, resulting in $F F_{\text {critical }}=w / w_{l}=0.69$. Assuming similar strain relaxation and heat dissipation, above such $F F_{\text {critical }}, I_{d}$ of the Tri-anode would be larger than the Planar at this voltage. Figure 7 (b) presents the calculated $F F_{\text {critical }}$ for the Tri-anode versus $V$. The $F F_{\text {critical }}$ is firstly small because of the smaller $V_{\text {on }}$ of the Tri-anode compared to the Planar, and after reaching a maximum value of $\sim 0.73$, it decreases due to the suppressed self-heating and trench conduction. Therefore, by increasing the $F F$ from 0.45 to 0.73 for the Tri-anode, its absolute output current normalized by device width would exceed that of the Planar. In other words, above $F F_{\text {critical }}$ the sidewall contact, superior heat management and trench conduction overcomes

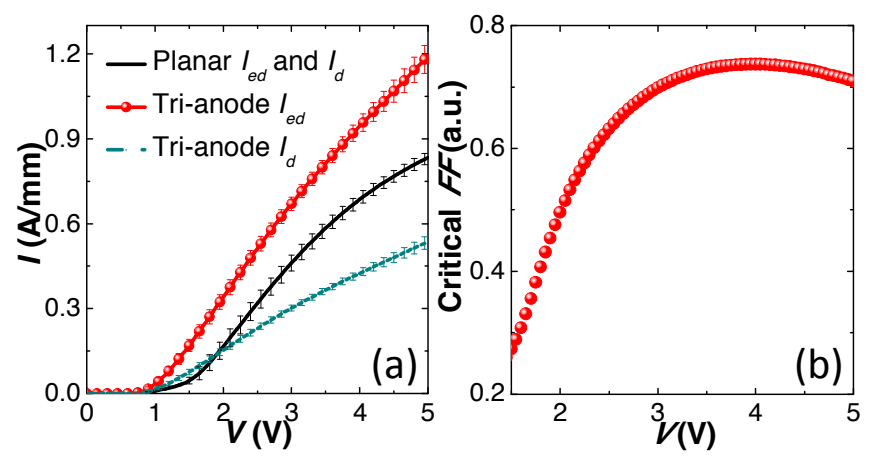

Fig. 7. (a) Current densities of the SBDs by different normalizations and (b) calculated critical $F F$ versus $V$ for the Tri-anode. 
the partial removal of the 2DEG. Considering that $w$ and the length of the nanowire will impact the $F F_{\text {critical }}$, further studies of dependence of $F F_{\text {critical }}$ on $w$, and their impact on output and thermal characteristics of the nanowire devices will be of great importance.

\section{CONCLUSION}

In conclusion, a hybrid tri-anode structure was investigated for $\mathrm{AlGaN} / \mathrm{GaN}$ SBDs in this work, which resulted in a reduction of $V_{\text {on }}$ from $1.43 \mathrm{~V}$ to $0.95 \mathrm{~V}$ and over 3 orders of magnitude lower reverse leakage current. The output current density normalized by effective anode width, and output linearity were enhanced by the integrated tri-gate transistors with trench conduction, which also largely diminished self-heating. We investigated the factors contributing to the electrical and thermal improvements in performance. Based on these results, we estimated the critical $F F$ above which the partial removal of the $2 \mathrm{DEG}$ in the hybrid tri-anode can be overcome. In addition to the improved on-state performance and the significant reduction of the off-state leakage current by the nanowire geometry, this work shows the potential of future nanowire devices presenting larger absolute output current than counterpart planar devices.

\section{ACKNOWLEDGMENT}

The authors would like to thank D. Gachet in Attolight AG and staff in the Center of MicroNanoTechnology at EPFL for technical support and valuable discussions.

\section{REFERENCES}

[1] W. Sito, Y. Takada, M. Kuraguchi, K. Tsuda, I. Omura, T. Ogura, and H. Ohashi, "High breakdown voltage AlGaN-GaN power-HEMT design and highg current density switching behavior", Electron Devices, IEEE transactions on, vol. 50, pp. 2528-2531, 2013.

[2] M. L. Schuette, A. Ketterson, B. Song, E. Beam, T. -M. Chou, M. Pilla, H. -Q, Tserng, X. Gao, S. Guo, P. J. Fay, H. G. Xing, and P. Saunier, "Gate-recessed integraed E/D GaN HEMT technology with $f_{T} / f_{\max }>300$ GHz", IEEE Electron Device Letters, vol. 34, pp. 741-743, 2011.

[3] T. Huang, Z. J. Liu, X. Zhu, J. Ma, X. Lu and K. M. Lau, "DC and RF performance of gate-last AlN/GaN MOSHEMTs on $\mathrm{Si}$ with regrown source/drain", Electron Devices, IEEE transactions on, vol. 60, pp 3019-3024, 2013.

[4] E. Matioli, B. Lu, and T. Palacios, "Ultralow Leakage Current AlGaN/GaN Schottky Diodes With 3-D Anode Structure", Electron Devices, IEEE transactions on, vol. 60, pp. 3365-3370, 2013.

[5] W. J. Ha, S. Chhajed, S. J. Oh, S. Hwang, J. K. Kim, J.-H. Lee, and K.-S. Kim, "Analysis of the revers leakage current in AlGaN/GaN Schottky barrier diodes treated with fluorine plasma", Applied Physics Letters, vol. 100, pp. 132104, 2012.

[6] T. -F. Chang, C. -F. Huang, T. Y-. Yang, C. -W. Chiu, T. -Y. Huang, K. -Y. Lee, and F. Zhao, "Low Turn-on voltage dual metal AlGaN/GaN Schottky barrier diode", Solid-State Electronics, vol. 105, pp. 12-15, 2015.

[7] G. -Y. Lee, H. -H. Liu, and J. -I Chyi, "High-performance AlGaN/GaN Schottky Diodes with an AlGaN/AIN buffer layer", IEEE Electron Device Letters, vol. 32, pp. 1519-1521, 2011.

[8] H. Woo, Y. Jo, J. Kim, C. Roh, J. Lee, H. Kim, H. Im, C. Ha, and J. Park, "Effect of heating on electrical transport in AlGaN/GaN Schottky barrier diodes on Si Substrate", Current Physics Letters, vol. 14, pp. 98-102, 2014.

[9] T. Tamura, J. Kotani, S. Kasai, and T. Hashizume, "Nearly temperature-independent saturation drain current in a multi-mesa-channel
AlGaN/GaN high electron mobility transistor", Applied Physics Express, vol. 1, pp. 023001, 2008

[10] J. T. Asubar, Z. Yatabe, and T. Hashizume, "Reduced thermal resistance in $\mathrm{AlGaN} / \mathrm{GaN}$ multi-mesa-channel high electron mobility transistors", Applied Physics Letter, vol. 48, pp. 053510, 2015.

[11] S. Liu, Y. Cai, G. Gu, J. Wang, C. Zeng, W. Shi, Z. Feng, H. Qin, Z. Cheng, K. J. Chen, and B. Zhang, "Enhanced-mode operation of nanochannel array (NCA) AlGaN/GaN HEMTs", IEEE Electron Device Letters, vol. 33, pp. 354-356, 2012.

[12] K. Ohi, J. T. Asubar, K. Nishiguchi, and T. Hashizume, "Current Stability in Multi-Mesa-Channel AlGaN/GaN HEMTs", Electron Devices, IEEE transactions on, vol. 60, pp. 2997-3004, 2013.

[13] K. -S, Im, V. Sindhuri, Y. -W. Jo, D. -H. Jo, D. -H. Son, J. -H. Lee, S. Cristoloveanu, and J. -H. Lee, "Fabrication of AlGaN/GaN $\Omega$-shaped nanowire fin-shaped FETs by a top down approach", Applied Physics Express, vol. 8, pp. 066501, 2015.

[14] B. Lu, E. Matioli, and T. Palacios, "Tri-gate normally-off GaN power MISFET”, IEEE Electron Device Letters, vol. 33, pp. 360-362, 2012.

[15] C. -Y. Chen, and Y. -R. Wu, "Studying the short channel effect in the scaling of the AlGaN/GaN nanowire transistors", Journal of Applied Physics, vol. 113, pp. 214501, 2013.

[16] M. A. Alsharef, R. Granzner, and F. Schiwierz, "Theoretical Investigation of Trigate AlGaN/GaN HEMTs", Electron Devices, IEEE transactions on, vol. 60, pp. 3335-3341, 2013

[17] S. P. McAlister, J. A. Bardwell, S. Haffouz, and H. Tang, "Self-heating and the temperature dependence of the $\mathrm{dc}$ characteristics of $\mathrm{GaN}$ heterostructure field effect transistor", Journal of Vacuum Science \& Technology A, vol. 24, pp. 624-628, 2006.

[18] R. J. T. Simms, J. W. Pomeroy, M. J. Uren, T. Martin, and M. Kuball, "Channel Temperature Determination in High-Power AlGaN/GaN HFETs using Electrical Methiods and Raman Spectroscopy", Electron Devices, IEEE transactions on, vol. 55, pp. 478-482, 2008.

[19] Z. Yan, G. Liu, J. M. Khan, and A. A. Balandin, "Graphene quilts for thermal management of high power $\mathrm{GaN}$ transistors", Nature Communications, vol. 3, pp.827-1, 2012.

[20] Y. -H. Hwang, T. -S. Kang, F. Ren, S. J. Pearton, "Novel approach to improve heat dissipation of $\mathrm{AlGaN} / \mathrm{GaN}$ high electron mobility transistors with a $\mathrm{Cu}$ filled via under device active area", Journal of Vacuum Science \& Technology B, vol. 32, pp. 061202-1, 2014.

[21] M. Hiroki, K. Kumakura, Y. Kobayashi, T. Akasaka, T. Makimoto, and H. Yamamoto, "Suppression of self-heating effect in AlGaN/GaN high electrcon mobility transistors by substrate-transfer technology using h-BN", Applied Physics Letters, vol. 105, pp. 193509-1, 2014.

[22] M. J. Tadjer, T. J. Anderson, K. D. Hobart, T. I. Feygelson, J. D. Caldwell, C. R. Eddy, F. J. Kub, J. E. Butler, B. Pate, and J. Melngailis, "Reduced Self-heating in AlGaN/GaN HEMTs using Nanocrystalline Diamond Heat-Spreading Films", IEEE Electron Device Letters, vol. 33, pp. 23-25, 2012.

[23] N. Defrance, Y. Douvry, V. Hoel, J. -C. Gerbedoen, A. Soltani, M. Rousseau, J. C. De Jaeger, R. Langer, and H. Lahreche, "Thermal resistance of $\mathrm{AlGaN} / \mathrm{GaN}$ HEMTs on SopSiC composite substrates", Electronics Letters, Vol. 46, No. 13, 2010.

[24] W. Liu and A. A. Balandin, "Thermal Conduction in $\mathrm{Al}_{\mathrm{x}} \mathrm{Ga}_{1-\mathrm{x}} \mathrm{N}$ alloys and thin films", Journal of Applied Physics, Vol. 97, pp. 973710-1, 2005.

[25] S. Takashima, Z. Li, and T. P. Chow, "Sidewall Dominated Characteristics on Fin-gate AlGaN/GaN mos-Channel-HEMTs", Electron Devices, IEEE transactions on, Vol. 60, pp. 3025-3031, 2013.

[26] Y. -W. Jo, D. -H. Son, C. -H. Won, K. -S. Im, J. H. Seo, I. M. Kang, and J. -H. Lee, "AlGaN/GaN FinFET With Extremely Broad Transconductance by Side-wall Wet Etching”, IEEE Electron Device Letters, vol. 36, pp. 1008-1010, 2015

[27] K. -S. Im, C. -H. Won, Y. -W. Jo, J. -H. Lee, M. Bawedin, S Cristoloveanu, and J. -H. Lee, "High-Performance GaN-based Nanochannel FinFETS With/Without AlGaN/GaN heterostructure", Electron Devices, IEEE transactions on, vol. 60, pp. 3012-3018, 2013.

[28] C. Yadav, P. Kushwaha, S. Khandelwal, J. P. Duarte, Y. S. Chauhan, and C. Hu, "Modeling of GaN-Based Normally-Off FinFET", IEEE Electron Device Letters, vol. 35, pp. 612-614, 2014.

[29] M. Azize and T. Palacios, "Top-down fabrication of AlGaN/GaN nanoribbons", Applied Physics Letters, Vol. 98, pp. 042103, 2011.

[30] Z. Li, H. Naik, and T. P. Chow, "Modeling and experimental study of MOS channel mobility of etched GaN on silicon substrate", Physica Status Solidi C, Vol. 8, pp. 2433-2435, 2011.

(C) 2016 IEEE. Personal use of this material is permitted. Permission from IEEE must be obtained for all other uses, in any current or future media, including reprinting/republishing this material for advertising or promotional purposes, creating new collective works, for resale or redistribution to servers or lists, or reuse of any copyrighted component of this work in other works. 
[31] B. Lu, O. I. Saadat, and T. Palacios, "High-performance integrated dual-gate $\mathrm{AlGaN} / \mathrm{GaN}$ enhancement-mode transistor", IEEE Electron Device Letters, vol. 31, pp. 990-992, 2010.

[32] K. Y. Fu, "Mobility degradation due to the gate field in the inversion layer of MOSFET's," IEEE Electron Device Letters, vol. 3, pp. 292-293, 1982.

[33] G. Ghibaudo, "New method for the extraction of MOSFET parameters", Electronics Letters, vol. 24, pp. 543-545, 1988.

[34] P. D. Ye, B. Yang, K. K. Ng, J. Bude, G. D. Wilk, S. Halder and J. C. M. Hwang, "GaN metal-oxide-semiconductor high-electron-mobility-transistor with atomic layer deposited $\mathrm{Al}_{2} \mathrm{O}_{3}$ as gate dielectric", Applied Physics Letters, vol. 86, pp. 063501, 2005.

[35] K. Tang, W. Huang, T. P. Chow, "GaN MOS capacitors and FETs on plasma-etched GaN surfaces”, Journal of Electronic Materials, Vol. 38, pp. 523-528, 2009.

[36] T. Narita, D. Kikuta, N. Takahashi, K. Kataoka, Y. Kimoto, T. Uesugi, T. Kachi, and M. Sugimoto, "Study of etching-induced damage in GaN by hard X-ray photoelectron spectroscopy", Physica Status Solidi A, Vol. 208, pp. 1541-1544, 2011.

[37] Liu, J. Ma, T. Huang, C. Liu, and K. M. Lau, "Selective epitaxial growth of monolithically integrated GaN-based light emitting diodes with AlGaN/GaN driving transistors", Applied Physics Letters, Vol. 104, pp.091103-1, 2014.

[38] N. Ikeda, J. Li, S. Yoshida, "Normally-off operation power AlGaN/GaN HFET", Proceedings of 2004 International symposium on Power Semiconductor Devices \& ICs, pp. 369-372, 2004. 